\title{
Asyl-Verwaltung kraft Wissen \\ Die Herstellung von Entscheidungswissen \\ in einer Schweizer Asylbehörde
}

LAURA AFFOLTER

\section{Einleitung}

\begin{abstract}
»Die bürokratische Verwaltung bedeutet: Herrschaft kraft Wissen: dies ist ihr spezifisch rationaler Grundcharakter. Über die durch das Fachwissen bedingte gewaltige Machtstellung hinaus hat die Bürokratie (oder der Herr, der sich ihrer bedient), die Tendenz die Macht noch weiter zu steigern durch das Dienstwissen: die durch Dienstverkehr erworbenen oder >aktenkundigen Tatsachenkenntnisse.«

(WEBER 2005: 165F.)
\end{abstract}

Wissensgenerierung ist ein zentrales Charakteristikum von bürokratischen Institutionen. Bürokratien produzieren Wissen (Hoag 2011: 81). Sie eignen sich Wissen über ihr Gegenstandsgebiet an und formen dieses gleichzeitig mit (Heyman 2004: 490). Laut Weber ist das Wissen, auf dem die Macht von Bürokratien beruht, ein ganz spezifisches. Es ist ein »Wissen von zweierlei Art«: Fachwissen und Dienstwissen (1988: 351). Als Fachwissen definiert er das »durch Fachschulung erworbene [...] im weitesten Sinne des Wortes stechnische [...]« Wissen (ebd.). Dienstwissen stellt für ihn die »nur dem Beamten zugängliche Kenntnis der für sein Verhalten maßgebenden konkreten Tatsachen« dar (ebd.). Während Fachwissen prinzipiell für alle zugänglich ist, handelt es sich bei Dienstwissen 
meist um Wissen, das »nicht generell verfügbar« ist (Derlien/Böhme/Heindl 2011: 92).

Will man das Funktionieren von Bürokratien verstehen, verlangt dies nach einer Auseinandersetzung damit, wie sie Wissen produzieren und mobilisieren. In meinem Beitrag zeige ich dies am Beispiel von decision-making im Schweizerischen Asylverfahren durch das Staatssekretariat für Migration (SEM) auf. ${ }^{1}$ Aufbauend auf Webers Definition von »bürokratischem Wissen« argumentiere ich erstens, dass im Entscheidungsprozess ein ganz bestimmtes Wissen generiert wird, das ich pragmatisch »Entscheidungswissen« nenne. Spezifisch ist dem Entscheidungswissen, dass es immer ein klares Endprodukt zum Ziel hat und auf dieses hinführt. Dieses Endprodukt ist der fertige Asylentscheid. Zweitens argumentiere ich, dass im Entscheidungsprozess eine weitere Form von Wissen eine wichtige Rolle spielt. Ich nenne diese Form »professionelles Wissen«. Professionelles Wissen bezeichnet jenes Wissen, das zur Ausführung einer Arbeit - im SEM wesentlich das Fällen von Asylentscheiden - nötig ist, jedoch in keiner schriftlichen Form vorliegt. Ich verwende den Begriff sowohl dafür, was die Entscheider_innen im SEM aus Erfahrung wissen, als auch dafür, was die Entscheider_innen selbst als >Bauchgefühlく bezeichnen. ${ }^{2}$ Er steht für das >einfach wissen <, ohne dass man genau sagen könnte, warum man etwas weiß. Professionelles Wissen bildet einen Teil des institutionellen Habitus von Sachbearbeitenden, da es ein Wahrnehmungs-, Denk- und Handlungsschema darstellt, das sich die Sachbearbeitenden durch ihre Arbeit in der Institution angeeignet haben (vgl. Steiner 2001: 24, 41f.; Bourdieu 1976). Da Sachbearbeitende sich professionelles Wissen (unbewusst) durch die institutionelle Sozialisierung aneignen müssen, bedeutet dies, dass diese Form von Wissen nicht generell zugänglich ist. Ich verstehe professionelles Wissen deshalb als eine weitere Form von Dienstwissen im Sinne Webers. Steiner folgend argumentiere ich, dass professionelles Wissen (als Teil des institutionellen Habitus) als »Generierungsprinzip von Verhaltensstrategien « zu verstehen ist. Es »regelt die Praxis, ohne jedoch Regeln zu erfüllen [und es] ist dem Zweck angepasst, ohne Ziele anzuvisieren« (Steiner 2001: $25)^{3}$

1 Das SEM besteht aus mehreren Direktionsbereichen. Ich beschäftige mich in meiner Arbeit ausschließlich mit dem Direktionsbereich Asyl.

2 Die Rolle des >Bauchgefühls` für Asylentscheide wurde unter anderen von Fassin (2013), Jubany (2011), Kelly (2012), Macklin (1998) und Thomas (2009) thematisiert.

3 Mit dem institutionellen Habitus von Sachbearbeitenden setze ich mich in meiner Dissertation genauer auseinander. Ich argumentiere dort, dass es sich bei professionellem Wissen zwar um subjektives Wissen, jedoch nicht um individuelles Wissen handelt. 
In meinem Beitrag gehe ich der Frage nach, wie im Asylverfahren Entscheidungswissen generiert wird. Ich argumentiere dabei, dass Entscheidungswissen das Produkt des Zusammenwirkens von Fachwissen in Form von Länder- und Gesetzeskenntnissen und Dienstwissen in Form von Aktenkundigkeit und professionellem Wissen ist. Dieses Argument leite ich im dritten Teil des Artikels her. Damit das Vorgehen in den Entscheidungsprozessen für die Leser_innen nachvollziehbar wird, zeige ich im zweiten Teil des Artikels die rechtlichen und institutionellen Bedingungen für decision-making im SEM auf.

Der Beitrag basiert auf den Analysen des Datenmaterials aus meiner laufenden Dissertationsforschung, worin ich mich mit der praktischen Entscheidungstätigkeit und im Speziellen mit der Praxis von Glaubhaftigkeitsbeurteilungen im SEM auseinandersetze. Zwischen 2013 und 2015 habe ich in verschiedenen Zentren und Sektionen des SEM ethnographische Forschung durchgeführt. Während meiner Aufenthalte in diesen verschiedenen Teams habe ich die Sachbearbeitenden bei ihrer täglichen Arbeit begleitet; ich habe Asylanhörungen beigewohnt, den Sachbearbeitenden beim Verfassen der Entscheide über die Schulter geschaut, mit ihnen >Fälle ২ diskutiert, an Sektionssitzungen teilgenommen und ich war auch in den Kaffeepausen dabei. Während dreier Wochen konnte ich außerdem an einer internen Ausbildung für neue Mitarbeitende teilnehmen. Meine Beobachtungen und Notizen zu informellen Gesprächen habe ich in Forschungstagebüchern festgehalten. Die Anhörungsprotokolle von `Fällen`, die ich während meiner Aufenthalte in irgendeiner Form begleitet habe, durfte ich kopieren und in anonymisierter Form für mein Forschungsprojekt verwenden, genauso wie die Entscheide zu diesen Gesuchen. Des Weiteren habe ich zu zwei zufällig gesetzten Daten alle Asyldossiers erhalten, über die an den betreffenden Tagen entschieden wurde und durfte daraus Entscheide, Anhörungsprotokolle und andere Dokumente kopieren. Schließlich habe ich semi-strukturierte Interviews mit 25 Sachbearbeitenden geführt, die ich aufgenommen und transkribiert habe. Die Analyse dieses Datenmaterials basiert auf dem Grounded-Theory-Ansatz nach Glaser und Strauss (1967). Ich habe mein Datenmaterial zuerst offen codiert, daraus analytische Kategorien geschaffen und die Beziehungen zwischen diesen Kategorien untersucht (vgl. Bryman 2011: 387ff.). Des Weiteren habe ich sowohl während der Forschung als auch während des Auswertungsprozesses analytische Memos geschrieben (vgl. Hammersley/Atkinson 2007: 150ff.).

Die Kollektivität von professionellem Wissen versuche ich in meiner Dissertation empirisch aufzuzeigen. 


\section{Asylverwaltung im SEM - Der Entscheidungskontext}

Wer in der Schweiz Asyl beantragt, kommt zuerst in eines der fünf Empfangsund Verfahrenszentren (EVZ) nahe der Landesgrenzen und beim Flughafen in Zürich. ${ }^{4}$ Dort findet eine erste kurze Befragung, eine sogenannte Befragung zur Person (BzP), statt, in der die Personalien und der Reiseweg erfragt und die Asylsuchenden gebeten werden, ihre Asylgründe kurz zu schildern. Wochen, Monate oder Jahre danach folgt entweder in einem der EVZ oder in der SEMZentrale in Bern die eigentliche Anhörung zu den Asylgründen. In einzelnen Fällen wird noch eine ergänzende Anhörung durchgeführt. Oft ist es die befragende Person dieser zweiten Anhörung, die den Asylentscheid fällt und ihn zum Kontrollieren und Visieren der Sektionschefin oder dem Sektionschef übergibt. ${ }^{5}$ Der Verwaltungsprozess beginnt also mit dem Erfassen der Personalien der gesuchstellenden Person beim Eintreffen in ein EVZ und endet - zumindest erstinstanzlich - damit, dass die sektionsleitende Person den Entscheid mit einer Unterschrift bestätigt und dieser der gesuchstellenden Person bzw. ihrer Rechtsvertretung zugestellt wird. Dazwischen geschehen jedoch noch einige Schritte.

\subsection{Die Arbeit im SEM}

$\mathrm{Zu}$ den Aufgaben der Sachbearbeitenden in den EVZ gehört es, BzP zu führen und $\mathrm{zu}$ protokollieren, Asylsuchende $\mathrm{zu}$ ihren Asylgründen anzuhören ${ }^{6}$ und Asylentscheide zu schreiben. Die Sachbearbeitenden in der Zentrale führen kei-

4 Ich beziehe mich im Folgenden auf das Verfahren, wie es im Moment noch in den fünf Empfangs- und Verfahrenszentren und in der Zentrale in Bern zur Anwendung kommt. Seit Januar 2014 gibt es in Zürich einen Testbetrieb, in welchem ein beschleunigtes Verfahren durchgeführt wird (https://www.bfm.admin.ch/bfm/de/home/ aktuell/news/2015/2015-02-16.html vom 08.04.15).

5 Andere Anhörungen werden von sogenannten >Poolies« durchgeführt. Die Bezeichnung >Poolies $<$ leitet sich davon ab, dass diese Personen zu einem Pool von Befragenden gehören, die im Stundenlohn arbeiten und keine regelmäßigen Arbeitszeiten haben. >Poolies` führen Anhörungen durch, treffen aber selbst keine Entscheide. Nach Anhörungen durch >Poolies` gehen die Asyldossiers mit dem Wortprotokoll der Anhörung an Sachbearbeitende des SEM, welche die Entscheide fällen.

6 Diese protokollieren sie nicht selbst, denn hier ist neben dem/der Gesuchsteller_in, dem/der Dolmetscher_in und der Hilfswerksvertretung auch ein/e Protokollführer_in anwesend. 
ne BzP durch, dafür haben die meisten von ihnen eine Federführung inne. Federführungen gibt es sowohl für die einzelnen Herkunftsländer als auch für bestimmte Themen, wie zum Beispiel geschlechtsspezifische Verfolgung oder Familienzusammenführung. Die Federführungen sind zuständig für die unité de doctrine (Einheitlichkeit der Entscheidungspraxis), also dafür, dass Gesuche von Asylsuchenden aus gleichen Ländern bzw. mit einem ähnlichen sogenannten Risikoprofil gleich behandelt werden. Das bedeutet nicht, dass die federführenden Personen alle diese Fälle selber behandeln. Sie erstellen und aktualisieren jedoch auf der Basis von wegweisenden (Grundsatz-)Urteilen des Bundesverwaltungsgerichts (BVGer), von Länderinformationen, die sie von den Länderreferentinnen und Länderreferenten ${ }^{7}$ erhalten, sowie von Praxisbestimmungen der Amtsleitung die jeweilige »Asyl- und Wegweisungspraxis«, kurz APPA genannt. APPAs sind eine Art amtsinterner Richtlinien, welche die Asyl- und Wegweisungspraxis in Bezug auf ein bestimmtes Land bzw. auf eine bestimmte Gruppe von Personen aus dem jeweiligen Herkunftsland definieren. ${ }^{8}$ Die federführenden Personen fungieren des Weiteren als Ansprechpersonen für die anderen Sachbearbeitenden bei Fragen, welche die Entscheidungspraxis betreffen.

\subsection{Die rechtlichen Grundlagen}

Das Schweizerische Asylgesetz (AsylG) und das Bundesgesetz über die Ausländer und Ausländerinnen $(\mathrm{AuG})$ bilden die Basis, auf der die institutionelle Entscheidungspraxis generiert und Asylentscheide getroffen werden. Im Entscheidungsprozess müssen die Sachbearbeitenden eine Reihe von Fragen beantworten, die ich in der untenstehenden Grafik vereinfacht dargestellt habe.

7 Die Länderreferentinnen und Länderreferenten arbeiten in der Abteilung »Analysen und Services«. Ihre Aufgabe besteht darin, Einzelfallanfragen von Sachbearbeitenden zu beantworten, Berichte zu schreiben und ein Monitoring in den Ländern durchzuführen, für die sie zuständig sind. Sie sind verantwortlich für die internen Länderinformationen und Datenbanken und bieten ab und zu Ausbildungen zu einzelnen Ländern an. Sie treffen jedoch keine Asylentscheide und bestimmen die Asyl- und Wegweisungspraxis nicht.

8 Die APPAs sind immer gleich aufgebaut. Im ersten Teil wird die allgemeine Situation im Land erklärt, dann folgt ein Teil mit Anweisungen, was in der BzP beachtet bzw. geprüft werden soll und danach ein Teil mit Anweisungen zu den Anhörungen. Nach diesen Anweisungen sind mögliche Asylmotive aufgeführt und die daraus folgenden Entscheide. Das Gleiche gibt es auch in Bezug auf die (Un-)Zumutbarkeit des Wegweisungsvollzugs. Hierbei handelt es sich um sogenannte Risikoprofile. 
Abbildung 1: Schematische Darstellung des Entscheidungsverfahrens

$$
\underset{\text { Nein }}{\longrightarrow}
$$

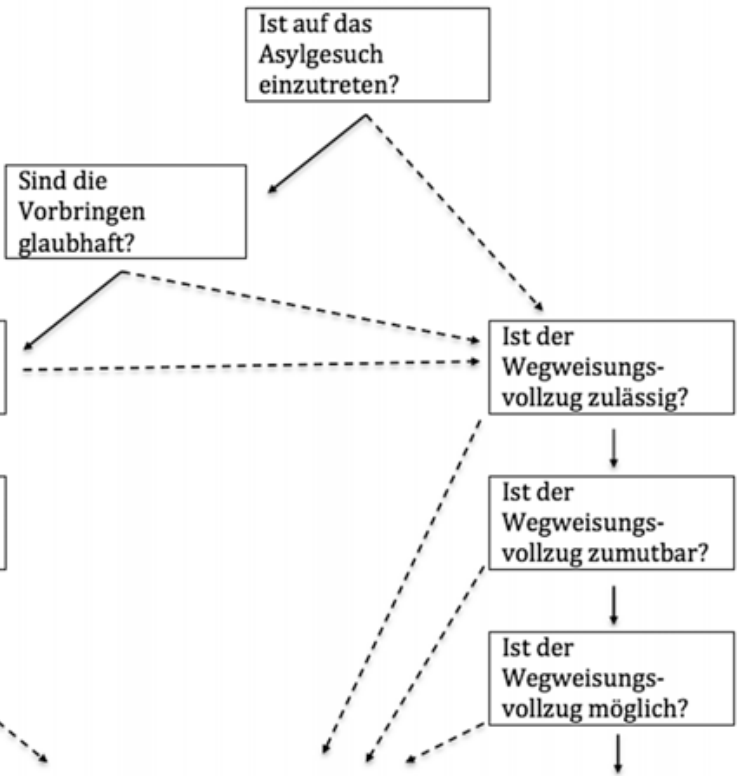

Asylgewährung

$$
\begin{array}{|l}
\text { Vorläufige } \\
\text { Aufnahme als } \\
\text { Flüchtling }
\end{array}
$$

Quelle: Eigene Darstellung auf der Grundlage des AsylG und AuG.

Ausschlaggebend dafür, ob eine Person Asyl erhält oder nicht, sind vor allem die Fragen nach der Flüchtlingseigenschaft gemäß Artikel 3 AsylG und nach der Glaubhaftigkeit gemäß Artikel 7 AsylG. Als Flüchtlinge gelten in der Schweiz Personen, »die in ihrem Heimatstaat oder im Land, in dem sie zuletzt wohnten, wegen ihrer Rasse, Religion, Nationalität, Zugehörigkeit zu einer bestimmten sozialen Gruppe oder wegen ihrer politischen Anschauungen ernsthaften Nachteilen ausgesetzt sind oder begründete Furcht haben, solchen Nachteilen ausgesetzt zu werden« (Art. 3, Abs. 1, AsylG). Dies gilt unter der Voraussetzung, dass keine Asylausschlussgründe (gemäß Art. 1 F Abkommen über die Rechtsstellung der Flüchtlinge und Art. 53 und 54 AsylG) ${ }^{9}$ vorliegen und die Vorbringen ${ }^{10}$

9 Artikel $1 \mathrm{~F}$ der Genfer Flüchtlingskonvention schließt Personen aus, die »ein Verbrechen gegen den Frieden, ein Kriegsverbrechen oder ein Verbrechen gegen die 
der asylsuchenden Person für glaubhaft befunden werden. »Glaubhaft gemacht ist die Flüchtlingseigenschaft, wenn die Behörde ihr Vorhandensein mit überwiegender Wahrscheinlichkeit für gegeben hält.« (Art. 7, Abs. 2, AsylG) Als unglaubhaft gelten die Vorbringen dann, »wenn sie in wesentlichen Punkten zu wenig begründet oder in sich widersprüchlich sind, den Tatsachen nicht entsprechen oder maßgeblich auf gefälschte oder verfälschte Beweismittel abgestützt werden« (Art. 7, Abs. 3, AsylG). Wir erkennen hier, dass sowohl die Flüchtlingseigenschaft als auch die Glaubhaftigkeit mit Wahrscheinlichkeiten begründet werden. So müssen die Sachbearbeitenden sowohl einschätzen, ob sie vorgebrachte vergangene Verfolgungen für überwiegend wahrscheinlich halten, als auch beurteilen, wie groß die Wahrscheinlichkeit zukünftiger Verfolgung ist.

Der Wortlaut »überwiegend wahrscheinlich« aus Artikel 7 AsylG wird von vielen Sachbearbeitenden dahingehend interpretiert, dass etwas - mathematisch betrachtet - zu 51 Prozent wahrscheinlich sein muss, um glaubhaft zu sein. Das wird einerseits als schwierig erachtet, weil Asylentscheide, so die Sachbearbeitenden, keine mathematischen Entscheide seien und die Bestimmung, ob etwas überwiegend wahrscheinlich sei, nach einer >subjektiven` Einschätzung verlange und andererseits, weil eine 51 prozentige Wahrscheinlichkeit Raum lasse für 49 Prozent Zweifel - was viele Sachbearbeitende als >ganz schön viel erachten. Die wahrgenommenen Schwierigkeiten hängen unter anderem mit dem sogenannten >niedrigeren Beweisstandard ‘ des Asylverfahrens beispielsweise im Vergleich zum Strafverfahren zusammen. Artikel 7 AsylG verlangt, dass die Flüchtlingseigenschaft nachgewiesen oder zumindest glaubhaft gemacht wird (Art. 7, Abs. 1, AsylG). Da Fluchtgründe oft materiell nicht bewiesen werden können, genügt in der Praxis meist die Glaubhaftmachung. Dies bedeutet einer-

Menschlichkeit im Sinne der internationalen Vertragswerke begangen haben«, die »ein schweres nichtpolitisches Verbrechen außerhalb des Aufnahmelandes begangen haben, bevor sie dort als Flüchtling aufgenommen wurden« oder die »sich Handlungen zu Schulden kommen ließen, die den Zielen und Grundsätzen der Vereinten Nationen zuwiderlaufen« (Art. 1 F Abkommen über die Rechtstellung der Flüchtlinge). Das Schweizerische Asylgesetz gewährt Flüchtlingen kein Asyl, »wenn sie wegen verwerflicher Handlungen dessen unwürdig sind oder wenn sie die innere oder die äußere Sicherheit der Schweiz verletzt haben oder gefährden« (Art. 53 AsylG) sowie »wenn sie erst durch ihre Ausreise aus dem Heimat- oder Herkunftsstaat oder wegen ihres Verhaltens nach der Ausreise Flüchtlinge im Sinne von Artikel 3 wurden« (Art. 54 AslyG).

10 Das Wort »Vorbringen« steht im Asylgesetz für die Aussagen von Asylsuchenden im Verfahren. 
seits, dass die mündlichen Aussagen von Asylsuchenden (bzw. die davon erstellten Wortprotokolle) die Basis bilden, auf der Asylentscheide getroffen werden. Andererseits bedeutet dieser >niedrigere $<$ Beweisstandard, dass praktisch alles als Beweis eingereicht werden kann und es gleichzeitig keine standardisierten Normen für die Interpretation dieser Beweise gibt (vgl. Kelly 2011: 185, 196) ${ }^{11}$

Im Asylverfahren müssen Sachbearbeitende Geschehnisse beurteilen, an denen sie selbst nicht teilgenommen haben und bei denen sie deshalb nie sicher wissen können, was wirklich passiert ist (vgl. Scheffer 2003: 425). Materielle Beweise und sogenannte Tatsachenkenntnisse kommen aus Sicht der Entscheider_innen einem an Sicherheit grenzenden Wissen am nächsten. ${ }^{12}$ Doch materielle Beweise fehlen häufig. Außerdem fehlt es den Sachbearbeitenden an Möglichkeiten und an Zeit, um solche Tatsachenkenntnisse zu generieren. ${ }^{13}$ Die meisten Sachbearbeitenden arbeiten nämlich unter großem Zeitdruck, da sie quantitative Ziele erreichen müssen, ebenso wie die einzelnen Asylabteilungen und Sektionen. ${ }^{14}$ Für die Glaubhaftigkeitsbeurteilung bedeutet dies laut einem Sachbearbeiter: »Im Strafverfahren gibt es Zeugen und jahrelange Prozesse. Und wir

11 Kelly (2011) zeigt auf, dass obwohl der Beweisstandard im Asylverfahren formell als niedrig gilt, er in der Praxis sehr hoch ist. Darauf gehe ich in meiner Dissertation näher ein.

12 Tatsachenkenntnisse basieren meist auf Dokumenten, denen ein Wahrheitswert zugeschrieben wird. So gilt es zum Beispiel als Tatsachenkenntnis, wenn man aufgrund von Medienberichten oder Länderreports weiß, wann ein bestimmtes Ereignis, wie beispielsweise eine Demonstration, stattgefunden hat und daraus ableiten kann, ob das, was die Person erzählt, >wahr ist oder nicht.

13 Gleichzeitig zeigt mein Datenmaterial, dass materielle Beweise in der Entscheidungspraxis sehr wohl eine Rolle spielen. So wurden 19 der 21 positiven Asylentscheide, die ich analysiert habe, zumindest teilweise mit materiellen Beweisen begründet. Ähnliches haben auch Good (2011) und Kobelinsky (2008) für die Verfahren in Großbritannien und Frankreich festgestellt.

14 Im SEM haben alle Mitarbeitenden quantitative Ziele, die sie erreichen sollten (genauso wie die einzelnen Asylabteilungen und Sektionen). Die Zahlen variieren je nach Stellenprozenten, Erfahrung im Amt, weiteren Funktionen etc. Während meiner Forschungsaufenthalte wurde in den meisten Fällen bei einer 100-Prozent-Stelle ein 〉Output` von ca. 18 bis 20 Entscheiden und vier bis fünf Anhörungen pro Monat verlangt. 
müssen in viereinhalb Stunden herausfinden, aufgrund einer Anhörung, ob etwas glaubhaft ist oder nicht.« (Interview T, meine eigene Übersetzung ${ }^{15}$ )

In Bezug auf die Einschätzung der begründeten Furcht vor zukünftiger Verfolgung wird durch institutionalisierte Praxis(-regeln) versucht, Wahrscheinlichkeitseinschätzungen vorweg zu nehmen. Dies geschieht einerseits dadurch, dass vergangene Verfolgung (sofern sie als glaubhaft und >aktuell beurteilt wird) als ein Indiz möglicher, zukünftiger Verfolgung gedeutet wird und andererseits durch die in den APPAs aufgeführten »Risikoprofile«. Die sogenannten Risikoprofile definieren Gruppen von Personen, von denen man annimmt, dass sie bei einer Rückkehr in ihr Heimatland verfolgt werden könnten. Je nach Land und Kontext können verschiedene Kriterien ausschlaggebend sein, ob man einer solchen Risikogruppe zugerechnet wird oder nicht. Solche Kriterien können beispielsweise Parteimitgliedschaften sein, der Wohnort vor der Ausreise, die Zugehörigkeit zu einer religiösen Gemeinschaft, das Alter etc. Für die Entscheidungspraxis bedeutet dies, dass der Bestimmung solcher Gruppenzugehörigkeiten eine wichtige Rolle zukommt.

Sowohl bestimmte Gruppenzugehörigkeiten als auch geltend gemachte vergangene Verfolgung werden jedoch nur dann als Indiz für zukünftige Verfolgung erachtet, wenn sie als glaubhaft bewertet werden. Die Glaubhaftigkeitsbeurteilung bzw. die rechtliche Argumentation mit dem Glaubhaftigkeitsartikel spielt insbesondere dann eine wichtige Rolle, wenn die Sachbearbeitenden für die Einschätzung der begründeten Furcht vor zukünftiger Verfolgung keine (>genauen`) Risikoprofile zur Verfügung haben. Je weniger man über ein Land wisse bzw. je willkürlicher ein Land funktioniere, desto schwieriger würde die Beurteilung der Gefahr durch zukünftige Verfolgung, wurde mir von Sachbearbeitenden mehrmals erklärt. In solchen >Fällen` sei es einfacher, mit dem Glaubhaftigkeitsartikel zu argumentieren:

»Zum Beispiel eine Mafia-Geschichte. Ist das asylrelevant oder nicht? [...] Weil [...] der Staat ist nicht unbedingt schutzfähig und es steckt zwar irgendwie kein Asylmotiv dahinter, aber [der Gesuchsteller] ist doch irgendwie einer Verfolgung ausgesetzt. Aber wenn du jetzt sagst, das alles ist unglaubhaft, dann sicherst du dich doch irgendwie ab." (Interview $\mathrm{R}$ )

15 Dies gilt auch für alle weiteren hier verwendeten Aussagen von SEM Sachbearbeitenden. Ich habe sowohl Aussagen aus dem Schweizerdeutschen als auch aus dem Französischen übersetzt. 
Im schweizerischen Asylverfahren wird die Mehrheit der Negativentscheide mit Unglaubhaftigkeit begründet. ${ }^{16}$ Dass die Glaubhaftigkeitsprüfung eine zentrale Rolle spielt, ergibt sich einerseits aus den rechtlichen Grundlagen. Die Grafik oben zeigt, dass die Glaubhaftigkeit von Asylvorbringen die Voraussetzung dafür ist, dass Gesuchstellende als Flüchtlinge anerkannt werden. Andererseits müsste die Glaubhaftigkeitsbeurteilung für negative Entscheide rechtlich gesehen nicht immer eine wichtige Rolle spielen. Für negative Entscheide reicht es nämlich, wenn nur eine der beiden Fragen »Sind die Vorbringen glaubhaft? « und »Erfüllt die Person die Flüchtlingseigenschaft?« mit »nein« beantwortet wird. Trotzdem wird in der Praxis meist mit dem Glaubhaftigkeitsartikel argumentiert und nicht mit dem Flüchtlingsbegriff. ${ }^{17}$ Meine These ist, dass dies einerseits mit dem verfahrensinhärenten Misstrauen zusammenhängt und andererseits damit, wie im Asylverfahren Entscheidungswissen hergestellt wird.

\section{Institutionelles Misstrauen}

»Aufgabe des Asylverfahrens ist es, unter den neu eintreffenden Asylsuchenden jene zu erkennen, die nach den beschriebenen Kriterien Anspruch auf Schutz haben. Viele Asylsuchende sind weder Flüchtlinge noch Kriegsvertriebene. [...] Sie suchen in der Schweiz einen besseren Platz zum Leben. [...] Für die Befragung durch die Behörden legen sich manche von ihnen eine dramatische Verfolgungsgeschichte zu. Sie hoffen, dadurch den Flüchtlingsstatus zu erlangen. Aus der Sicht des Betroffenen ist dieses Verhalten verständlich, aus asylrechtlicher Perspektive handelt es sich um einen Missbrauch des Asylverfahrens. Die Behörden müssen solche Gesuche möglichst rasch abweisen und die Wegwei-

16 Statistiken dazu, ob negative Entscheide alleine aufgrund von Artikel 7 oder von Artikel 3 AsylG bzw. durch eine Kombination von beiden begründet wurden, gibt es nicht, da solche Entscheide im System mit dem gleichen Code versehen werden. Doch im Empfinden aller meiner Gesprächspartner_innen kommen Artikel-7-Entscheide viel häufiger vor als reine Artikel-3-Entscheide. Auch im Handbuch »Asyl und Rückkehr« des SEM steht: »Die Mehrzahl der Ablehnungen von Asylgesuchen ist auf die fehlende Glaubhaftigkeit der Vorbringen der asylsuchenden Personen zurück zu führen.« (Staatssekretariat für Migration [SEM] o.J.: 7) Mein Dossier-Sample zeigt, dass von den 39 Negativentscheiden, die ich analysiert habe, in 27 Fällen unter anderem mit Artikel 7 AsylG argumentiert wurde.

17 Dies entspricht der Amtspraxis, zu der die Sachbearbeitenden angeleitet werden. 
sung konsequent vollziehen. Dadurch wird das Asylverfahren für arbeitsuchende AusländerInnen unattraktiv. ${ }^{18}$

Dies schreibt das SEM unter »Kernpunkte der Eidgenössischen Asylpolitik« auf seiner Webseite. Wir erkennen in diesem Auszug die Funktion der Sachbearbeitenden als »gatekeepers« oder »guardians of a common good « (Heyman 2009: 381; Hoag 2011: 82; Lipsky 2010: 4). Das »common good « ist in diesem Fall eine (vorläufige) Aufenthaltsberechtigung in der Schweiz. Sachbearbeitende können es vergeben, müssen es aber auch vor Missbrauch schützen. Des Weiteren erkennen wir auch die Annahme, dass viele der Asylsuchenden nicht schutzbedürftig seien und bei der Anhörung lügen würden, um Schutz zu erhalten. Diese Lügen müssten durch das Verfahren aufgedeckt werden. Viele Wissenschaftler_innen, die sich mit Asyl- und Immigrationsverfahren beschäftigen, beschreiben denn auch, dass Entscheidungsfindungen häufig von Misstrauen begleitet werden (vgl. Alpes/Spire 2014; Fassin/D’Halluin 2005; Kelly 2012). So wird Misstrauen - quasi als die Umkehrung von Naivität - als ein Zeichen von Professionalität gesehen (vgl. Alpes/Spire 2014: 269). ${ }^{19}$ Die Annahme, dass viele Asylgeschichten konstruiert seien, führt dazu, dass Anhörungen in der Praxis zu einer Suche nach (Un-)Wahrheiten werden (vgl. Anderson et al. 2014; Jubany 2011; Macklin 1998; Souter 2011).

Im Gegensatz zum Strafverfahren, in dem zunächst die Unschuldsvermutung gilt, geht es deshalb in Asylanhörungen darum, so lange zu fragen, bis einen die gesuchstellende Person von der >Wahrheit` der Geschichte überzeugt hat oder bis man genügend Argumente für einen negativen Asylentscheid hat, mit denen man wiederum andere überzeugen kann - in erster Linie die sektionsleitende Person und im Fall einer Beschwerde das Bundesverwaltungsgericht:

»A: [...] Und dann lasse ich die Person erzählen und prüfe die Glaubwürdigkeit.

F: In jedem Fall?

A: Ja, eigentlich schon. Also, das ist nicht, um böse zu sein, nicht, dass ich jetzt davon ausgehe, dass sie sowieso lügen, das nicht. Aber man möchte halt wissen, ob sie es wirklich erlebt haben. Und was sie davon wissen. [...] Teilweise merkt man schnell, dass es stimmt. [...] Dann hake ich zum Teil nicht mehr nach, man muss sie ja nicht noch mehr belasten. Und wenn es mir nicht glaubwürdig erscheint, dann hake ich

18 https://www.bfm.admin.ch/bfm/de/home/asyl/asyl/asylrecht.html vom 16.10.15.

19 Im SEM höre ich oft, dass man, wenn man neu im Amt sei, halt noch ein bisschen zu naiv sei - eine solche Naivität wird auch mir zugeschrieben. Erst die Erfahrung lehre einen, nicht mehr alles >einfach so< zu glauben. 
nach, bis ich entweder genügend Argumente habe für meinen Entscheid, genügend Widersprüche, oder bis er mich überzeugt hat, dass es doch stimmt.« (Interview A)

Die Aussagen des Sachbearbeiters verdeutlichen, dass nicht alle Geschichten per se in Zweifel gezogen werden - man kann auch schnell >merken`, dass etwas stimmt. Aber man muss es eben zuerst >merken jedem Fall eine Umkehrung der Unschuldsvermutung stattfindet. Denn anders kann die Glaubhaftigkeit gar nicht geprüft werden. Es ist eine Prüfung, die es selbst im Vertrauensfall braucht, denn es kann nur dann positiv entschieden werden, wenn man die nötigen Argumente dazu hat. Und die müssen erst produziert werden.

\section{Bürokratische Entscheidungslogik und die Herstellung von Entscheidungswissen}

»Entscheiden, das ist unser Handwerk. [...] Das ist unser Kerngeschäft [...]. Das heißt, wir sind im Prinzip wie Cäsar in der Arena, der so macht [Daumen hoch] oder so macht [Daumen runter], wir sagen ja oder nein. Es gibt noch so gewisse Grautöne dazwischen, aber letztlich sagen wir ja oder nein, das ist der Entscheid. Und der Entscheid muss so gewissen Kriterien entsprechen. [Kriterien], die man auflisten kann, die sich aus Recht und Praxis ergeben und dann hat es irgendwo dazwischen immer noch so das Bauchkriterium.«(Interview V)

Die Sachbearbeiterin spricht in diesem Zitat drei Punkte an, die ich in diesem Teil des Artikels diskutiere. Erstens beschreibt die Sachbearbeiterin die Form von Entscheiden: jeder Entscheid muss eindeutig mit »ja» oder »nein« beantwortet werden. Zweitens wird klar, dass jeder Asylentscheid mit Gesetzeskriterien begründet werden muss. Drittens spricht die Sachbearbeitende die Rolle des >Bauchgefühls` für das Fällen von Asylentscheiden an. Ersteres verbinde ich mit der von Handelman definierten »bürokratischen Logik«. Auf die letzten beiden Punkte gehe ich im Zusammenhang mit der Herstellung von Entscheidungswissen ein.

Handelman definiert bürokratische Logik als die Schaffung linearer Formen der Klassifikation (2004: 6). Ohne solche Formen der Klassifikation könnten Bürokratien gar nicht bestehen (vgl. Handelman 1995: 280). Die taxonomischen Klassifikationsschemata, die Handelman beschreibt, bestehen aus Kategorien, die hierarchisch angeordnet sind und sich eindeutig voneinander abgrenzen. Entweder gehört etwas in eine Kategorie oder es wird eindeutig davon ausge- 
schlossen. Auf einem solchen Schema beruht auch das decision-making im SEM, wie man der Grafik im vorhergehenden Teil entnehmen kann. Die Grafik zeigt die vier Rechtskategorien, denen die Gesuchstellenden durch das Verfahren eindeutig zugeordnet werden müssen. Die Zuordnung geschieht über eine Reihe hierarchisch angelegter Fragen, die alle eindeutig mit »ja« oder »nein« beantwortet werden müssen. Eine Antwort dazwischen gibt es nicht.

Es reicht jedoch nicht aus, »Ja-« oder »Nein-« Entscheide zu treffen. Jede Entscheidung muss auch begründet werden. Bei negativen Entscheiden geschieht dies verfahrensöffentlich; Gesuchstellende erhalten ein Schreiben mit den Gründen für den negativen Asylentscheid. Positive Entscheide hingegen werden nur intern begründet. Damit will man verhindern, dass Gesuchstellende lernen, wie man ein erfolgreiches Asylgesuch stellt. Sowohl positive als auch negative Asylentscheide werden dadurch begründet, dass Gesetzeskriterien mit aktenkundigen Tatsachen verknüpft werden. ${ }^{20}$ Im Verlauf des Verfahrens müssen also erstens aktenkundige Tatsachen und zweitens Verknüpfungen mit dem Gesetz hergestellt werden. Das Wissen, das zu diesem Zweck generiert wird, nenne ich Entscheidungswissen.

Entscheidungswissen ist jenes Wissen, das zu einem Endprodukt, dem fertigen Asylentscheid, führt. Es ist charakteristisch für die bürokratische Verwaltung. Ich argumentiere, dass Entscheidungswissen durch das Zusammenspiel von Fach- und Dienstwissen generiert wird. Unter Fachwissen verstehe ich im untersuchten Kontext die Länder- und Gesetzeskenntnisse von Sachbearbeitenden. $^{21}$ Dienstwissen bezieht sich, wie Weber (2005: 165f.) schreibt, auf aktenkundige Tatsachenkenntnisse, die durch das Verfahren erzeugt werden. Im Asylverfahren sind dies alle Schriftstücke, die im Dossier abgelegt werden: die auf jeder Seite mit einer Unterschrift der asylsuchenden Person bestätigten Anhörungsprotokolle, materielle Beweise, Gutachten von sogenannten Expertinnen und Experten etc. Über Weber (ebd.) hinausgehend argumentiere ich, dass in bürokratischen Entscheidungsprozessen noch eine weitere Form von Dienstwissen existiert und zur Generierung von Entscheidungswissen beiträgt: professionelles Wissen. Unter professionellem Wissen verstehe ich das, was die Sachbearbei-

20 In der Praxis geschieht dies in beide Richtungen, wie ich im Folgenden aufzeige. Sachbearbeitende gehen teilweise von Tatsachen aus und suchen nach passenden Gesetzeskriterien oder sie gehen umgekehrt von bestimmten Gesetzeskriterien aus und suchen im Asyldossier nach passenden Tatsachen.

21 Mit Gesetzeskenntnis meine ich sowohl die Kenntnis relevanter Gesetzesartikel, die für Asylentscheide verwendet werden, als auch die Kenntnis formalisierter institutioneller Praxisregeln, wie man sie vor allem in den APPAs findet. 
tenden selbst jeweils als >Bauchgefühl bezeichnen. Professionelles Wissen ist jedoch nicht nur eine Intuition, wie das Wort >Bauchgefühl« suggeriert. Vielmehr ist es geprägt durch das Fachwissen, das Erfahrungswissen und die Entscheidungsroutinen, welche die Sachbearbeitenden erarbeitet haben.

Im Folgenden zeige ich zunächst am Beispiel der Herkunftsbestimmung die Verknüpfung von Länderwissen und aktenkundigen Tatsachen auf. Danach lege ich anhand des Entscheidungskriteriums »Widersprüche« dar, wie im und durch das Asylverfahren aktenkundige Tatsachen hergestellt werden und mit Recht in Verbindung gebracht werden. Schließlich untersuche ich, welche Rolle professionelles Wissen für die Generierung von Entscheidungswissen spielt. Ich zeige auf, dass professionelles Wissen eine richtungsweisende Funktion hat, jedoch im fertigen Asylentscheid unsichtbar gemacht wird.

\subsection{Länderkenntnisse für die Herkunftsprüfung}

Die Ermittlung der (sozialen) Herkunft von Gesuchstellenden gehört zu den ersten Schritten im Asylverfahren und ist richtungsweisend für das weitere Vorgehen. In erster Line gilt es dabei ’herauszufinden<, aus welchem Land der/die Gesuchsteller_in kommt bzw. in welchem Land er oder sie sogenannt hauptsozialisiert wurde. Aber auch andere biographische Merkmale, wie zum Beispiel das Alter der Person, ihr Bildungsgrad, ihr genauer Wohnort im Herkunftsland etc. werden über das Verfahren ermittelt. All diese `Herkunftskriterien` sind entscheidend für die Zuordnung zu bestimmten Risikoprofilen (oder umgekehrt für den Ausschluss aus bestimmten Risikoprofilen), sowohl in Bezug auf die Flüchtlingseigenschaft als auch auf den Vollzug der Wegweisung.

Am einfachsten und schnellsten geht die Herkunftsbestimmung anhand von Identitätsdokumenten. ${ }^{22}$ Da materielle Beweise oft fehlen, wird in der Praxis versucht, die Herkunft von Gesuchstellenden zu eruieren anhand von Fragen nach dem, was Scheffer die »Mitgliedschaftskompetenz« nennt (Scheffer 2001: 146f.). ${ }^{23}$ Anhand dieser Fragen soll bestimmt werden, ob die von den Gesuchs-

22 Wenn Identitätsdokumente eingereicht werden, gibt es für die Sachbearbeitenden drei Handlungsmöglichkeiten: Sie können die Dokumente als echt anerkennen, sie können die Dokumente auf ihre Echtheit hin überprüfen lassen oder sie können die Beweisdokumente als untauglich bewerten. Letzteres kommt beispielsweise dann zur Anwendung, wenn Gesuchstellende Kopien von Identitätsdokumenten einreichen, insbesondere wenn diese nicht sie selbst, sondern zum Beispiel Familienmitglieder betreffen.

23 In den 50 bisher von mir analysierten Dossiers wurden in 16 Fällen originale Identitätsdokumente eingereicht. In vier Fällen haben die Sachbearbeitenden ein Dokumen- 
tellenden angegebene Herkunft >stimmt oder nicht . Die Herkunftsprüfung wird in der Regel in der Befragung zur Person in den Empfangs- und Verfahrenszentren durchgeführt. Gefragt wird danach, was jemand, der oder die aus einem bestimmten Land oder Ort kommt, >wissen müsste`, wie man in diesem Auszug aus einem BzP-Protokoll erkennt:

$\gg$ F: Wie lautet die Telefonvorwahl von Marokko?

A: 00212 .

F: Wie heißt der König von Marokko?

A: (GS lächelt) Das ist Mohammed der VI. Ich bin ein echter Marokkaner.

F: Wie heißt seine Frau, die Königin?

A: Ich weiß, dass sie aus Fes stammt. Als er geheiratet hat, war ich nicht in Marokko.

F: Wie heißen die umliegenden Ortschaften [von] Kneitra?

A: Kneitra liegt ca. $40 \mathrm{~km}$ von Rabat entfernt. In Kneitra gibt es einen amerikanischen Stützpunkt. Die umliegenden Ortschaften heißen Sidi Yahia, Sidi Suleiman und Sidi Kassem.« (Auszug aus einem BzP Protokoll)

In diesem Fall hat der Gesuchsteller die Herkunftsprüfung bestanden; er hat seine Mitgliedschaftskompetenz bewiesen. Die Sachbearbeiterin hat die Antworten des Gesuchstellers anhand ihres eigenen Länderwissens bewertet. Ihr Länderwissen hat sie sich selbst angeeignet: durch das Lesen von internen Länderreports, durch das, was sie von anderen Gesuchstellenden in der Anhörung erfahren hat, durch eigene Internetrecherchen, das Studieren von Landkarten etc. Wenn die Sachbearbeitenden nach der Prüfung der Mitgliedschaftskompetenz die Herkunft von Asylsuchenden immer noch anzweifeln, kann eine Lingua-Analyse in Auftrag gegeben werden, die den Sozialisierungsort der Gesuchstellerin oder des Gesuchstellers ermitteln soll. ${ }^{24}$ Manchmal stellen die Sachbearbeitenden auch Botschaftsanfragen und bitten die Leute vor Ort, zu ermitteln, ob eine Person

tengutachten erstellen lassen; die Echtheit des Dokumentes wurde in allen vier Fällen bestätigt.

24 Lingua-Analysen werden auf der Webseite des SEM folgendermaßen definiert: »LINGUA ermittelt mit Hilfe von externen, unabhängigen Experten den Sozialisationsraum der Gesuchsteller und hält die Resultate der Ermittlungen in einem Gutachten fest. Die LINGUA-Gutachten basieren auf einer linguistischen Analyse der Sprache und auf einer landeskundlich/kulturellen Analyse des Wissens der asylsuchenden Person.« (https://www.bfm.admin.ch/bfm/de/home/asyl/asylverfahren/drei_beispiele/ analysen.html vom 21.04.15) 
beispielsweise tatsächlich im angegeben Quartier gelebt hat. ${ }^{25}$ Die LinguaGutachten verkörpern sowohl Fachwissen als auch Dienstwissen. Sie basieren auf dem Länder- und Sprachwissen von sogenannten Expertinnen und Experten. Gleichzeitig gelten Gutachten wie auch Botschaftsantworten als aktenkundige Tatsachen, die für Entscheidungsbegründungen verwendet werden können.

\subsection{Widersprüche als aktenkundige Tatsachen}

Meine Analyse der Asyldossiers zeigt, dass Widersprüche das meist verwendete Unglaubhaftigkeitskriterium in negativen Asylentscheiden sind: so wurde in 21 von 27 >Unglaubhaftigkeitsentscheiden` die Unglaubhaftigkeit unter anderem mit dem Kriterium »Widersprüche« begründet. In Anbetracht dessen, dass die Entscheider_innen im SEM unter hohem Zeitdruck arbeiten, argumentiere ich, dass die häufige Verwendung des Kriteriums »Widersprüche « damit zusammenhängt, dass solche Argumente einfach und schnell zu produzieren sind und keiner weiteren Abklärungen (wie zum Beispiel Botschaftsanfragen) bedürfen. Denn Widersprüche >findet man in den Protokollen, sie werden durch die Anhörungen selbst produziert.

Die häufige Verwendung des Kriteriums drückt sich auch in dessen Beliebtheit aus. In meinen Interviews habe ich den Sachbearbeitenden Karteikarten gegeben, auf denen die Kriterien aus den Textbausteinen für negative Entscheide mit Artikel 7 AsylG standen. ${ }^{26}$ Ich habe die Sachbearbeitenden gebeten, die Karten in eine Rangordnung zu bringen; beginnend mit dem Kriterium, das sie wenn möglich - am liebsten für die Begründung von Unglaubhaftigkeit verwenden, absteigend bis zum Kriterium, das sie am wenigsten gerne verwenden. Im Anschluss daran habe ich mit den Sachbearbeitenden über ihr Ranking gespro-

25 Wie genau die Mitgliedschaftskompetenz geprüft wird, also wie viele und wie detaillierte Fragen dabei gestellt werden, und ob noch weitere Abklärungen wie LinguaAnalysen oder Botschaftsanfragen getätigt werden, hängt von verschiedenen Faktoren ab. Eine wichtige Rolle spielt dabei das angegebene Herkunftsland selbst. Zum Teil bestehen institutionelle Vorgaben betreffend der Herkunftsbestimmung von Asylsuchenden aus bestimmten Ländern. In anderen Fällen wissen die Sachbearbeitenden aus Erfahrung, wie genau die Herkunft zu prüfen ist.

26 Für die Argumentation in Negativentscheiden mit Artikel 7 AsylG stehen den Sachbearbeitenden Textbausteine zu folgenden Kriterien zur Verfügung: gefälschte Beweismittel, mangelnde Substantiierung, nachgeschobene Vorbringen, nicht mehr geltend gemachte Vorbringen, Tatsachenwidrigkeit, untaugliche Beweismittel, widersprechen der allgemeinen Erfahrung, widersprechen der Logik des Handelns, Widersprüche. 
chen. Die allermeisten Sachbearbeitenden haben das Kriterium »Widersprüche« an die erste oder zweite Stelle gesetzt (hinter »Tatsachenwidrigkeit «). ${ }^{27}$ Begründet wurde dies meist damit, dass Entscheide mit Widersprüchen einfach seien und dass man diese gut belegen könne, da man Fakten habe, genauso wie bei gefälschten Beweisen oder Tatsachenwidrigkeiten. ${ }^{28}$

Die Argumentation mit Widersprüchen basiert auf dem Aussagenabgleich. Dabei werden Vergleiche zwischen verschiedenen Aussagen gezogen: zwischen den Aussagen, welche die gesuchstellende Person in der BzP gemacht hat und denen in der Anhörung, zwischen verschiedenen Aussagen in der Anhörung selbst sowie zwischen Aussagen verschiedener Personen, die verwandt oder zusammen geflüchtet sind. Die Grundannahme für solche Vergleiche ist, dass eine Person, die etwas erlebt hat, bzw. mehrere Personen, die das Gleiche erlebt haben, immer wieder das Gleiche erzählen würden. Das Verfahren an sich bietet dadurch, dass jede_r Asylsuchende mindestens zweimal befragt wird, und dass verwandte Personen, wie zum Beispiel Ehepartner, getrennt voneinander befragt werden, mehrere Abgleich-Möglichkeiten. Andere Abgleich-Möglichkeiten entstehen durch das Vorgehen in den Anhörungen und die Fragen, die dabei gestellt werden.

Obwohl es keinen Fragenkatalog für Anhörungen gibt und die Befrager_innen ihre eigenen Fragen entwickeln, sind das Vorgehen in den Anhörungen und die Fragen, die gestellt werden, meist sehr ähnlich. ${ }^{29}$ Die Anhörung beginnt nach einer kurzen Einführung, in der die Anwesenden (in ihrer Funktion) vorgestellt werden und die gesuchstellende Person auf ihre Rechte und Pflichten hingewiesen wird, mit der Frage nach Beweisdokumenten. Danach folgen häufig Fragen zur Familie und zum Leben im Herkunftsland. Der nächste Teil zu den Asylgründen beginnt meist mit einer oder mehreren offenen Fragen, wie zum

27 Während die meisten meiner Gesprächspartner_innen »Widersprüche« als eines der besten und einfachsten Kriterien bewerteten, fanden andere, dass die Argumentation mit Widersprüchen problematisch sei, weil es kaum möglich sei, eine Geschichte zweimal gleich zu erzählen. Sie setzten das Kriterium deshalb im Ranking auf eine der hintersten Stellen und kritisierten die häufige Verwendung des Kriteriums in Asylentscheiden.

28 Siehe Fußnote 12.

29 Dies hat wohl unter anderem damit zu tun, dass die Sachbearbeitenden voneinander lernen, welche Fragen man stellen könnte. Dies insbesondere beim Anfangscoaching, bei dem sie Anhörungen von anderen beiwohnen. Aber auch später haben sie immer wieder mal mit Protokollen anderer Befragungen zu tun. Des Weiteren lassen sich in der Maske für das Anhörungsprotokoll und in den APPAs Beispielfragen finden. 
Beispiel »Weshalb haben Sie (Land XY) verlassen und in der Schweiz Asyl beantragt?«, gefolgt von gezielten W-Fragen und geschlossenen Fragen. Zum Schluss werden die Gesuchstellenden meist mit sogenannten Widersprüchen konfrontiert. Dieses Vorgehen vergleichen viele Entscheider_innen mit einem Trichter oder dem Zuziehen einer Schlinge. So geht es bei Anhörungen oft darum zu testen, ob sich die gesuchstellende Person in Widersprüche $>$ verstrickt oder nicht<.

Sachbearbeitende erachten sowohl das Stellen von offenen Fragen als auch das Stellen von zusammenhängenden Fragen als hilfreiche Strategien, um Widersprüche raufzudecken`. So erklärten mir einige Sachbearbeitende, dass sich die Gesuchstellenden - sofern die Geschichte nicht >wahr sei - viel eher sin Widersprüche verstricken « würden, wenn man zuerst eine offene Frage stelle und die Gesuchstellenden dann lange und frei erzählen lasse. Das Gleiche passiere auch, wenn man sich-aufeinander-beziehende Fragen stelle, denn diese könnten die Gesuchstellenden oft nicht widerspruchslos beantworten. Durch solche Fragestrategien werden aktenkundige Tatsachen erzeugt, die sich mit dem Kriterium »Widersprüche« verbinden lassen. Wie dies in der Praxis geschieht, stelle ich anhand >eines Falles` des Sachbearbeiters Bernard dar. ${ }^{30}$

Es handelt sich dabei um ein Gesuch eines jungen Eritreers, der als Asylgrund geltend machte, aufgrund einer unterstellten Beziehung zu einem Regimekritiker zweimal von Soldaten zuhause aufgesucht worden zu sein: Einmal hätten die Soldaten ihn befragt und geschlagen, beim zweiten Mal sei sein Haus durchsucht worden. Daraufhin habe er seine Heimatstadt Stadt Barentu verlassen. Bernard hat das Gesuch abgelehnt und den Entscheid hauptsächlich mit dem Kriterium »Widersprüche« begründet. ${ }^{31}$ Viele der von ihm im Entscheid aufgeführten Widersprüche beziehen sich auf Zeitangaben und die chronologische Abfolge von Geschehnissen:

»Während Sie [der Entscheid ist direkt an den Gesuchsteller gerichtet] in Ihrer freien Erzählung vorbringen, zwischen der ersten behördlichen Befragung und der Hausdurchsuchung durch das Militär seien 20 Tage bis ein Monat vergangen [vgl. Akte X, S. Y], machen Sie später geltend, zwischen der ersten behördlichen Befragung und der Hausdurch-

30 Aus Anonymisierungsgründen habe ich verschiedene Identifikationsmerkmale des Sachbearbeiters und der gesuchstellenden Person (Name, Herkunftsland, Geschlecht etc.) geändert. Auch die Auszüge aus dem Asylentscheid und den Anhörungsprotokollen habe ich abgeändert.

31 Der Mann erhält jedoch eine vorläufige Aufnahme, da er aufgrund seines militärtauglichen Alters einem Risikoprofil im APPA entspricht. 
suchung seien lediglich zwei bis drei Tage vergangen [vgl. Akte XX, S. Y]. Gleich darauf sagen Sie zudem aus, Ihr Haus sei einige Wochen nachdem Sie aufgefordert worden seien in den Militärdienst einzurücken, durchsucht worden [vgl. Akte XX, S. Z ].« (Auszug aus dem Asylentscheid)

Bernard ist über die Anhörung zu diesem Argument gekommen. Wie üblich hat Bernard den Anhörungsteil zu den Asylgründen mit einer offenen Frage begonnen: »Gut, dann können Sie mir jetzt frei erzählen, was Ihnen persönlich in Eritrea geschehen ist und warum Sie Eritrea dann verlassen haben.« (Auszug aus dem Anhörungsprotokoll) In seiner Antwort sagte der Gesuchsteller unter anderem:

»Es kamen etwa zwei, drei bewaffnete Personen zu mir. Sie haben mir Fragen gestellt. [...] Diese Leute haben mich dann verbal beleidigt. Dann haben sie mich geschlagen. Was danach passierte, weiß ich nicht. [...] Man ließ uns etwa 20 Tage bis einen Monat in Ruhe. [...] Die Soldaten haben dann unser Haus durchsucht.« (Auszug aus dem Asylentscheid)

Später in der Anhörung begann Bernard dem Gesuchsteller geschlossene Fragen und gezielte W-Fragen zu stellen. Darunter waren auch solche, welche die Befragung und die Hausdurchsuchung durch die Soldaten betrafen:

$» F$ : Nur eine kleine Frage zur Bestätigung: Wenn ich Sie richtig verstanden habe, kamen die Behörden zweimal zu Ihnen nach Hause, das erste Mal wurden Sie befragt und man hat Sie geschlagen und beim zweiten Mal wurde das Haus beschlagnahmt. Stimmt das so?

A: Ja. Sie haben gewusst, dass ich Kontakt zu X hatte. Die Regierung hat überall Spione.

F: Wie viel Zeit ist zwischen diesen Ereignissen etwa vergangen?

A: Kurze Zeit, etwa zwei, drei Tage. Man wollte ja von mir unbedingt wissen, was mir X gesagt hatte.

F: Können Sie sich auch noch an das Datum erinnern, an dem Ihr Haus beschlagnahmt wurde und Sie Barentu verlassen haben?« (Auszug aus dem Befragungsprotokoll)

Und schließlich sprach er den Gesuchsteller am Ende der Anhörung auf einzelne Widersprüche an:

»F: Sie haben heute ausgesagt, nach der ersten behördlichen Befragung hätten Sie etwa 20 Tage Ruhe gehabt. Später haben Sie dann gesagt, zwischen den zwei Befragungen seien zwei oder drei Tage vergangen.

A: Ja. 
F: Was stimmt denn jetzt?

A: Es waren etwa zwei oder drei Tage. Wenn ich das letzte Mal von etwa 20 Tagen sprach, dann war damit die Zeitspanne gemeint, als ich Barentu verlassen habe. Es gab viele Probleme, deshalb kann ich mich nicht mehr an alles genau erinnern.« (Auszug aus dem Anhörungsprotokoll)

Am Ende der Anhörung war Bernard überzeugt, dass diese (und andere) Aussagen des Gesuchstellers unglaubhaft waren. Der Gesuchsteller hatte ihn nicht vom Gegenteil überzeugen können. Der >Fall endete damit so, wie es Bernard schon nach dem Lesen des BzP-Protokolls vermutete hatte. Denn Bernard waren bereits beim Lesen des Protokolls ein paar >Unstimmigkeiten dies üblich ist, hatte er die Anhörung deshalb in eine bestimmte Richtung vorbereitet und im Vorfeld Fragen notiert, von denen er glaubte, dass sie - sollte die Geschichte tatsächlich unglaubhaft sein - aktenkundige Widersprüche erzeugen würden. Letztlich ist genau das geschehen.

\subsection{Von professionellem Wissen zu Entscheidungswissen}

Wie Bernard wissen Entscheider_innen meist schon früh, wie der Entscheid ausfallen wird. Die Schwierigkeit liegt denn auch nicht darin, Entscheidungen zu fällen, sondern diese zu begründen. Erst mit der Begründung ist das Entscheidungswissen komplett. Erst damit kommt man nämlich zu einem Endprodukt, dem fertigen Asylentscheid. Dass die Entscheidungsfindung an sich von den Sachbearbeitenden als einfach betrachtet wird, hat mit professionellem Wissen zu tun.

Professionelles Wissen trägt zur Herstellung von Entscheidungswissen bei, indem es die Richtung vorgibt (oder zumindest mitbestimmt), in welche befragt wird. ${ }^{32}$ Bernard zum Beispiel hat Fragen vorbereitet, die auf Widersprüche abzielen. Umgekehrt war ich ein anderes Mal bei einer Anhörung dabei, in welcher die Sachbearbeiterin gänzlich auf die Glaubhaftigkeitsprüfung verzichtete, weil sie nach dem Lesen des BzP-Protokolls bereits überzeugt war, dass die Geschichte glaubhaft sei. Als ich sie fragte, warum dies für sie so klar sei, meinte sie: »Ich schaue mir das Protokoll an und ich weiß es.« (Auszug aus dem Forschungstagebuch) Außerdem hätte der Gesuchsteller sehr detailliert und in einer logischen und chronologischen Weise gesprochen. Und er hätte Dinge gesagt, die auf sein Heimatland zutreffen würden.

32 Die Richtung kann sich im Verlauf des Verfahrens auch ändern. 
Dass professionelles Wissen eine wichtige Bedeutung hat für die Richtungsbestimmung von Entscheiden, zeigt sich auch darin, dass Sachbearbeitende es bevorzugen, Entscheidungen zu treffen, wenn sie die Person selbst angehört haben. Einerseits, weil sie selbst die Fragen stellen und so einfacher Argumente für den Entscheid generieren können. Anderseits empfinden es viele als hilfreich, die asylsuchende Person selbst zu sehen und zu erleben. Man bekomme dann einen >persönlichen Eindruck` von der Person. Der `persönliche Eindruck ‘ bezieht sich nicht nur auf die verbalen Aussagen der asylsuchenden Person, sondern auch auf deren non-verbales Verhalten während der Anhörung. Zwar wird mir von den Sachbearbeitenden in den Interviews jeweils entsprechend der offiziellen Norm gesagt, das Verhalten und die gezeigten Emotionen der Gesuchstellenden seien vollkommen irrelevant. Wenn ich jedoch mit Sachbearbeitenden, zum Beispiel während Anhörungspausen, konkrete `Fälle ২ bespreche, wird häufig auf Verhalten und Emotionen der Gesuchstellenden Bezug genommen, um Entscheidungen oder erste Ideen einer Entscheidungsrichtung zu begründen.

»Es ist mehr wie ein Gefühl. Und nachher suche ich im Text danach« (Interview U), erklärte mir ein Sachbearbeiter sein Vorgehen bei der Glaubhaftigkeitsbeurteilung. Diese Aussage verdeutlicht, dass professionelles Wissen nicht nur die Richtung mitbestimmt, in die befragt wird, sondern auch die Richtung, in welcher während des Schreibprozesses nach Argumenten gesucht wird. Gesucht wird dabei vor allem in den Anhörungsprotokollen. Das folgende Beispiel von Sachbearbeiterin Manuela zeigt dies deutlich ${ }^{33}$ :

»Ich sitze neben Manuela an ihrem Pult und schaue ihr beim Verfassen des Entscheides zu. Es geht um eine Frau aus Sri Lanka, und eigentlich steht der Entscheid für Manuela bereits fest: die Frau bekommt kein Asyl, aber eine vorläufige Aufnahme. Für Manuela ist >der Fall klar unglaubhaft, denn die Vorbringen der Frau sind ihrer Auffassung nach völlig unsubstantiiert. Gleichzeitig bemerkt Manuela, dass die Gesuchstellerin auf jede Nachfrage hin etwas mehr hinzufügt. Eine reine Argumentation mit Unsubstantiiertheit findet sie deshalb schwierig. Manuela bedauert, dass in den Aussagen der Gesuchstellerin keine Widersprüche drin sind, denn Widersprüche würden die Argumentation von Unglaubhaftigkeit viel einfacher machen. Zur Sicherheit hat sich Manuela deshalb noch das Protokoll des Bruders der Frau angeschaut. Einfach um zu schauen, ob es ihr Gefühl bestätigt oder nicht. In diesem Fall wurde es bestätigt. Manuela sagt mir, dass es wohl einen kombinierten Entscheid [Entscheid, in welchem mit Artikel 3 und Artikel 7 AsylG argumentiert wird] geben werde, denn mit der Unglaubhaftigkeit alleine hätte sie zu wenig. Aber vielleicht, meint sie, komme es ja dann beim Schreiben ganz anders.

33 Siehe Fußnote 30. 
Vor ihrem Computer sitzend, beginnt sie nun, den Sachverhalt aufzuschreiben. Dann wählt sie zwei Textbausteine aus, einen zu Artikel 3 und einen zu Artikel 7 AsylG, nämlich den der mangelnden Substantiierung. Nun beginnt sie in den Protokollen nach Textstellen zu suchen, welche dieses Argument belegen. Immer wieder springt sie zwischen dem Sachverhalt und den Argumenten hin und her. Während sie weiter nach Argumenten für Unsubstantiiertheit sucht, fallen ihr einige Widersprüche auf, insbesondere zwischen den Protokollen der Gesuchstellerin und denen ihres Bruders. Sie notiert sich diese und nimmt noch den Textbaustein zu »Widersprüchen« hinzu. Während sie schreibt, erklärt sie mir, dass dieser Vergleich hier wichtig sei, weil sie sonst nicht genügend Argumente hätte. Sie glaubt aber, dass andere wohl mit dem Argument der Unsubstantiiertheit zufrieden gewesen wären. Aber sie würde sich damit nicht sicher genug fühlen. Weil sie dem Gesuchsteller nun noch auf schriftlichem Weg das rechtliche Gehör gewähren muss, um diese Widersprüche überhaupt verwenden zu dürfen, legt sie das Dossier weg und wendet sich anderen Aufgaben zu.« (Auszug aus dem Forschungstagebuch)

Das Beispiel zeigt, dass der Fall für Manuela schon zu Beginn des Schreibprozesses klar war: die Aussagen der Frau waren unglaubhaft. Manuela fand jedoch zunächst nicht genügend aktenkundige Tatsachen, die ihr >Gefühl bestätigten und mit denen sie sich sicher genug fühlte, denn sie wusste, dass sie mit ihren Argumenten sowohl ihren Vorgesetzten, als auch - im Fall einer Beschwerde das BVGer überzeugen musste. Somit war ihr Entscheidungswissen zu Beginn inkomplett. Dies änderte sich mit dem Einbezug des Dossiers des Bruders. Durch den Vergleich der beiden Dossiers fand Manuela Argumente für das Kriterium »Widersprüche«. Es gelang ihr, aktenkundige Tatsachen mit Recht zu verknüpfen: das Entscheidungswissen war komplett. ${ }^{34}$ Zwar wusste Manuela nicht genau, was dieser Frau in Sri Lanka alles widerfahren war. Sie vermutete nämlich, dass die Frau ihre wahren Ausreisegründe nicht erzählt habe. Was ihre Gründe waren und warum die Gesuchstellerin diese nicht erzählt hatte, wusste sie nicht. Sie konnte darüber nur spekulieren. Sie musste es aber auch nicht wissen, denn für einen eindeutigen, legitimen Asylentscheid war ihr Entscheidungswissen genug.

34 Manuela verwendete in ihrem endgültigen Entscheid mehrere Kriterien aus Artikel 7 und Artikel 3 AsylG. Es waren aber vor allem die Widersprüche, die ihr beim Schreiben die Sicherheit gaben, den Entscheid so zu treffen, wie sie es für richtig befand. 


\section{Fazit}

Entscheidungswissen ist stets eindeutiges Wissen. Es erzeugt die begründete »Ja-« oder »Nein-« Beantwortung hierarchisch angelegter Fragen. Dadurch werden Asylgesuche klar einer von vier Rechtskategorien zugeordnet. Entscheidungswissen ist also nicht Wissen um des Wissens willen. Es zielt immer auf ein Verwaltungsprodukt ab: den fertigen Asylentscheid. Das bedeutet, dass Entscheidungswissen nur so komplex und ambivalent sein kann, wie es noch eindeutige »Ja-« oder »Nein-« Antworten zulässt. Gleichzeitig kann es Wissenslücken bestehen lassen, wenn sie für die Entscheidungsfindung irrelevant sind. Dies zeigt das Beispiel der Sachbearbeiterin Manuela. Sie glaubte, dass die Gesuchstellerin andere Gesuchgründe hatte als diejenigen, die sie in den Anhörungen geltend gemacht hatte. Welche das waren, wusste sie nicht, musste sie für den Asylentscheid aber auch gar nicht wissen. Es hätte in der Verantwortung der Gesuchstellerin gelegen, ihr diese Gründe zu schildern. ${ }^{35}$ Eine andere Form von Wissenslücken ergibt sich durch die Wahrscheinlichkeitseinschätzungen, auf denen Artikel 3 und Artikel 7 AsylG beruhen: Bei Artikel 3 geht es um die Einschätzung, wie begründet die Furcht vor zukünftiger Verfolgung ist, und Artikel 7 verlangt nach einer Einschätzung, wie wahrscheinlich das Vorhandensein der Flüchtlingseigenschaft ist. Im Wort »Wahrscheinlichkeit« sind Wissenslücken bereits inhärent, denn Wahrscheinlichkeit kann immer nur auf partiellem Wissen beruhen. So kann die Zukunft nicht mit hundertprozentiger Sicherheit vorausgesagt werden, und sicher wissen, was >wirklich nicht dabei war, kann man auch nicht (vgl. Scheffer 2003: 425). Solche Wissenslücken sind jedoch nur so lange ein praktisches Entscheidungsproblem, wie kein Entscheidungswissen an deren Stelle gesetzt wird.

»In all legal judgments, recognized uncertainty has to be transformed into practical certainty«, schreibt Kelly (2011: 185). Dies gilt auch für das Entscheidungsverfahren im SEM. Die Ausgangslage ist aufgrund der eben genannten Wissenslücken meist eine ungewisse. Durch das Verfahren muss eine »praktische Gewissheit« geschaffen werden; eine Gewissheit, die rechtlich legitimierba-

35 Asylsuchende Personen unterstehen der sogenannten Mitwirkungspflicht nach Artikel 8 AsylG. Diese verlangt, dass Gesuchsteller_innen »ihre Identität offenlegen, vorhandene Reise- und Identitätsausweise und allfällig vorhandene Beweismittel abgeben oder, soweit vorhanden und beschaffbar, sich um ihre Beibringung bemühen « (Schweizerische Flüchtlingshilfe SFH 2009: 79). Des Weiteren müssen die Gesuchstellenden »bei der Befragung detailliert und vollständig [...] begründen, weshalb sie in der Schweiz um Asyl« nachsuchen (ebd.). 
re Entscheide ermöglicht. Diese praktische Gewissheit ist Entscheidungswissen. In meinem Beitrag habe ich aufgezeigt, wie Entscheidungswissen im Verlauf des Verfahrens hergestellt wird. Welche Rolle die verschiedenen Wissensformen dabei spielen, wie sie genau zusammenspielen und sich gegenseitig bedingen, ist fallspezifisch und kann in dieser Komplexität nicht dargestellt werden. Allgemein kann jedoch gesagt werden, dass Entscheidungswissen sich in schriftlicher Form durch die Verknüpfung von Gesetzeskriterien mit aktenkundigen Tatsachen äußert. Diese Verknüpfungen sowie die aktenkundigen Tatsachen selbst müssen jedoch erst über das Verfahren hergestellt werden, wie die Beispiele der Sachbearbeitenden Bernard und Manuela zeigen. Dies geschieht, indem Gesuchstellende auf bestimme Art und Weise befragt werden und indem zusätzliche Abklärungen getätigt werden, wie zum Beispiel Lingua-Analysen, Länderconsultings, Botschaftsabklärungen etc. Die Richtung, in welche aktenkundige Tatsachen generiert werden, sowie deren Verknüpfung mit Gesetzeskriterien werden durch professionelles Wissen geprägt. Entscheidungswissen ist somit mehr als das, was gegen außen sichtbar wird. Es beruht auch auf einer >gefühlten Sicherheit<.

Am Beispiel von decision-making im SEM habe ich aufzuzeigen versucht, wie im Asylverfahren verschiedene Formen von Wissen produziert und mobilisiert werden. Ich habe dabei Webers Definition des Dienstwissens eine weitere Form hinzugefügt: professionelles Wissen. Weber ging es jedoch nicht >nur darum, »bürokratisches Wissen« zu definieren. Vielmehr argumentierte er, dass bürokratische Verwaltung »Herrschaft kraft Wissen« bedeutet (Weber 2005: 165f.). Dies habe ich auch für das SEM gezeigt. Die Macht des Entscheidungswissens, das $>$ Fälle< überhaupt erst entscheid- und kategorisierbar macht, liegt darin, dass es nicht für alle zugänglich ist. Zwar könnte sich theoretisch Jede_r Länder- und Gesetzeskenntnisse aneignen. Der Einfluss, den Gesuchstellende und andere in das Verfahren involvierte Personen auf die Herstellung von aktenkundigen Tatsachen und deren Verwendung haben, ist jedoch gering. Ferner bleibt professionelles Wissen gegen außen hin stets ganz verborgen.

\section{Literatur}

Alpes, Maybritt Jill/Spire, Alexis (2014): »Dealing with Law in Migration Control: The Powers of Street-level Bureaucrats at French Consulates«, in: Social \& Legal Studies 23(2), S. 261-274.

Anderson, Jessica/Hollaus, Jeannine/Lindsay, Annelisa/Williamson, Collin (2014): »The culture of disbelief. An ethnographic approach to understand- 
ing an under-theorised concept in the UK asylum system. «, in: Working paper series no. 102, Oxford: Refugee Studies Centre.

Bourdieu, Pierre (1976): Entwurf einer Theorie der Praxis auf der ethnologischen Grundlage der kabylischen Gesellschaft, Frankfurt a.M.: Suhrkamp.

Bryman, Alan (2011): Social Research Methods, Oxford: Darin.

Derlien, Hans-Ulrich/Böhme, Doris/Heindl, Markus (2011): Bürokratietheorie: Einführung in eine Theorie der Verwaltung (= Studienskripten zur Soziologie), Wiesbaden: VS Verlag für Sozialwissenschaften.

Fassin, Didier (2013): »The Precarious Truth of Asylum«, in: Public Culture 25(1), S. 39-63.

Fassin, Didier/D'Halluin, Estelle (2005): »The Truth from the Body. Medical Certificates as Ultimate Evidence for Asylum Seekers«, in: American Anthropologist 107(4), S. 597-608.

Glaser, Barney G./Strauss, Anselm L. (1967): The discovery of grounded theory: strategies for qualitative research, Chicago: Aldine.

Good, Anthony (2011): »Witness statement and credibility assessments in the British asylum courts«, in: Livia Holden (Hg.), Cultural Expertise and Litigation, Abingdon, New York: Routledge, S. 94-122.

Hammersley, Martyn/Atkinson, Paul (2007): Ethnography. Principles in practice. Third edition, London/New York: Routledge.

Handelman, Don (1995): »Comments on >Putting Power in the Anthropology of Bureaucray: The Immigration and Naturalization Service at the MexicoUnited States Border` by Josiah McC. Heyman«, in: Current Anthropology 36(2), S. 280-281.

Handelman, Don (2004): Nationalism and the Israeli State. Bureaucratic Logic in Public Events, Oxford/New York: Berg.

Heyman, Josiah McC (2004): »The Anthropology of Power-Wielding Bureaucracies«, in: Human Organization 63(4), S. 487-500.

Heyman, Josiah McC. (2009): »Trust, Privilege, and Discretion in the Governance of US Borderlands with Mexico«, in: Canadian Journal of Law and Society 24(3), S. 367-390.

Hoag, Colin (2011): »Assembling Partial Perspectives: Thoughts on the Anthropology of Bureaucracy«, in: PoLAR: Political and Legal Anthropology Review 34(1), S. 81-94.

Jubany, Olga (2011): »Constructing truths in a culture of disbelief. Understanding asylum screening from within«, in: International Sociology 26(1), S. 74-94.

Kelly, Tobias (2011): »The legalization of human rights and the protection of torture survivors. Asylum, evidence and disbelief«, in: Marie-Bénédicte 
Dembour/Tobias Kelly (Hg.), Are Human Rights for Migrants? Critical Reflections on the Status of Irregular Migrants in Europe and the United States, New York: Routledge, S. 184-200.

Kelly, Tobias (2012): »Sympathy and suspicion: torture, asylum, and humanity«, in: Journal of the Royal Anthropological Institute 18(4), S. 753-768.

Kobelinsky, Carolina (2008): »The Moral Judgement of Asylum Seekers in French Reception Centres«, in: Anthropology News 49(5), S. 5-11.

Lipsky, Michael (2010): Street-Level Bureaucracy. Dilemmas of the Individual in Public Services, New York: Russel Sage Foundation.

Macklin, Audrey (1998): Truth and Consequences: Credibility Determination in the Refugee Context. Conference paper, Ottawa: International Association of Refugee Law Judges.

Scheffer, Thomas (2001): Asylgewährung. Eine ethnographische Analyse des deutschen Asylverfahrens, Stuttgart: Lucius \& Lucius.

Scheffer, Thomas (2003): »Kritik der Urteilskraft. Wie die Asylprüfung Unentscheidbares in Entscheidbares überführt«, in: Jochen Oltmer (Hg.), Migration steuern und verwalten: Deutschland vom späten 19. Jahrhundert bis zur Gegenwart, Göttingen: Vandenhoeck \& Ruprecht, S. 423-458.

Schweizerische Flüchtlingshilfe SFH (Hg.) (2009): Handbuch zum Asyl- und Wegweisungsverfahren, Bern: Haupt.

Souter, James (2011): »A Culture of Disbelief or Denial? Critiquing Refugee Status Determination in the United Kingdom«, in: Oxford Monitor of Forced Migration 1(1), S. 48-59.

Steiner, Pascale (2001): Bourdieu lesen und verstehen (= Arbeitsblätter des Instituts für Ethnologie der Universität Bern, Nummer 19), Bern: Institut für Ethnologie.

Thomas, Robert (2009): »Refugee Roulette: A UK Perspective«, in: Jaya RamjiNogales/Andrew I. Schoenholtz/Philip G. Schrag (Hg.), Refugee Roulette. Disparities in Asylum Adjudication and Proposals for Reform, New York/London: New York University Press, S. 164-186.

Weber, Max (1988): »Parlament und Regierung im neugeordneten Deutschland«, in: Johannes Winckelmann (Hg.), Gesammelte politische Schriften, Tübingen: J.C.B. Mohr (UTB).

Weber, Max (2005): Wirtschaft und Gesellschaft, Nürnberg: Zweitausendeins. 


\section{Internetquellen}

Abkommen über die Rechtsstellung der Flüchtlinge vom 28. Juni 1951: http://www.unhcr.ch/fileadmin/user_upload/unhcr_ch/Mandat/GFK_und_Pr otokoll.pdf vom 24.04.15.

Asylgesetz vom 26. Juni 1998 (Stand 1. Oktober 2015): https://www.admin.ch/ opc/de/classified-compilation/19995092/index.html vom 28.12.15.

Bundesgesetz über die Ausländerinnen und Ausländer (Stand 1. Oktober 2015): https://www.admin.ch/opc/de/classified-compilation/20020232/index.html vom 10.02.16.

https://www.bfm.admin.ch/bfm/de/home/aktuell/news/2015/2015-02-16.html vom 08.04.15.

https://www.bfm.admin.ch/bfm/de/home/asyl/asyl/asylrecht.html vom16.10.15. https://www.bfm.admin.ch/bfm/de/home/asyl/asylverfahren/drei_beispiele/analy sen.html vom 21.04.15.

Staatssekretariat für Migration (SEM): Handbuch Asyl und Rückkehr. Artikel C5 Der Nachweis der Flüchtlingseigenschaft. https://www.bfm.admin.ch/ dam/data/bfm/asyl/verfahren/hb/c/hb-c5-d.pdf vom 24.04.15. 
Bereitgestellt von | Universitätsbibliothek Bern

Angemeldet

Heruntergeladen am | 06.06.18 15:08 